\title{
Lasing from all-polymer microcavities
}

\author{
G Canazza ${ }^{1}$, F Scotognella ${ }^{2,3}$, G Lanzani ${ }^{2,3}$, S De Silvestri ${ }^{4}$, \\ M Zavelani-Rossi ${ }^{4}$ and D Comoretto ${ }^{1}$
}

1 Dipartimento di Chimica e Chimica Industriale, Università degli Studi di Genova, via Dodecaneso 31,
I-16146 Genova, Italy
${ }^{2}$ Dipartimento di Fisica, Politecnico di Milano Piazza L. da Vinci 32, I-20133 Milano, Italy
${ }^{3}$ Center for Nano Science and Technology @ PoliMi, Istituto Italiano di Tecnologia via G. Pascoli 70/3,
I-20133 Milano, Italy
${ }^{4}$ Dipartimento di Fisica, IFN-CNR, Politecnico di Milano Piazza L. da Vinci 32, I-20133 Milano, Italy

E-mail: margherita.zavelani@polimi.it and davide.comoretto@unige.it

Received 4 September 2013

Accepted for publication 13 January 2014

Published 12 February 2014

\begin{abstract}
We report on the laser action in a microcavity where both the dielectric mirrors and the active material have a macromolecular nature, resulting in a full plastic laser device. Distributed Bragg reflectors (DBRs) are prepared by spin-coating of polyvinylcarbazole and cellulose acetate orthogonal solutions and the active layer consists of a highly fluorescent conjugated polymer poly(9,9-dioctylfluorenyl-2,7-diyl-co-1,4-benzo-(2,1'-3)-thiadiazole) (F8BT) spun film. A quality factor in the range $80-180$ is achieved and the cavity mode is carefully tuned on the peak of the F8BT amplified spontaneous emission spectrum. Under pulsed optical pumping, laser emission is obtained with a surprisingly low lasing threshold $\left(<20 \mu \mathrm{J} \mathrm{cm}{ }^{-2}\right)$ for a full plastic DBR optical cavity. This result opens a simple and cheap way to obtain a new class of polymer lasers.
\end{abstract}

Keywords: polymer laser, polymer microcavities, polymer multilayers, polymer active devices

(Some figures may appear in colour only in the online journal)

\section{Introduction}

Since the first report of lasing action from conjugated polymers in solution [1], a considerable research effort has been made by the scientific community working in the field in order to improve all different facets involved in the process (photophysics, materials, and device structure). Concerning this latter item, different architectures such as distributed feedback lasers, distributed Bragg reflectors (DBR), photonic crystals and microcavities have been used to engineer the optical cavity architecture (see for instance paper [2-5] and references therein reported). Among the different lasing cavities, the planar microcavity is particularly interesting since it can be easily engineered, especially when using macromolecules, and it is suitable to be integrated in nano-photonics devices. It generally consists of a multilayered structure of inorganic oxides (silica, titania, zirconia, hafnia. . . ) possessing different refractive indices (acting as DBRs) embedding a structural defect containing the gain material [6]. This represents a very favorable solution when using semiconducting polymers as the active material because it allows an easy and low-cost fabrication method suitable for photonic devices. Moreover, it is more versatile than the microcavities in which the organic material is directly spun over an inorganic commercial high reflectivity DBR mirror and closed with a metallic mirror [7, 8] or with another inorganic DBR [9-16].

Microcavities are even more attractive if the DBR structure is prepared with polymer materials instead of traditional inorganic insulators, because all plastic, flexible devices can be envisaged. Different approaches have been developed, such as self-assembly of high molecular mass block-copolymers, or wide area polymer DBRs prepared by co-extrusion in a 
continuous melt processing $[17,18]$. However, the synthesis of block copolymers is complicated while co-extrusion can be applied only to a limited number of materials. A very powerful and cost effective method to prepare organic DBRs is spin-coating of polymer solutions. When polymers possessing different refractive indexes are solved in non-miscible (orthogonal) solvents, it is possible to prepare high quality DBRs by spinning alternate layers of the two materials. By changing solution viscosity and spin velocity, the photonic band gap can be tuned in different spectral positions and structural defects can be easily introduced [19-21]. Different devices of this kind have been proposed such as multilayers doped with a gain dye, where amplified spontaneous emission has been observed [22, 23], microcavities with a defect layer containing colloidal quantum dots [24, 25] or dye molecules [26, 27]. All polymer microcavities, where both the dielectric mirrors and the defect layer have an intrinsic macromolecular nature, were reported for the first time by some of us [28]. There, the DBRs were made of polystyrene and cellulose acetate while the defect layer of F8BT. Their optical properties were fully characterized by means of polarized angular-resolved transmittance and photoluminescence spectral measurements. The strong directional emission enhancement, observed under $\mathrm{CW}$ excitation, makes these structures promising for lasing.

In this paper we report on laser emission from an all plastic microcavity under pulsed optical pumping. By choosing higher dielectric contrast polymers, the DBR performance was improved in terms of reflectivity and bandwidth. Cellulose acetate and poly(vinylcarbazole) have been employed for the DBRs and the F8BT for the active layer, resulting in a high microcavity quality factor. A very low threshold has been observed for laser emission. The threshold value is lower than the ones reported for microcavities with organic DBRs and fluorophores or nanocrystal active media [24, 25, 29], and similar to those obtained with organic active media and inorganic DBRs [30, 31]. Lower threshold values have been reported only by using commercial high-reflectivity dielectric mirrors [32, 33], which however do not possess the flexibility of an all plastic device where both the active material and the optical cavity have a macromolecular nature.

\section{Experimental details}

Cellulose acetate (CA, $M_{\mathrm{w}} 61000$, refractive index $n=1.475$ at $600 \mathrm{~nm}$, from Sigma Aldrich) and poly(vinylcarbazole) (PVK, $M_{\mathrm{w}} 90$ 000, $n=1.675$, from Across Organics) have been employed to obtain high optical quality 1D photonic crystal and microcavity structures by dynamic spin-coating of polymer solutions in orthogonal solvents [23, 28, 34]. Cellulose acetate has been solved in 4-hydroxy-4-methyl2-penthanon (diacetone alcohol, from Sigma Aldrich) with a concentration about $24 \mathrm{~g} \mathrm{l}^{-1}$ while PVK was solved in toluene (Sigma Aldrich) at a similar concentration. F8BT was purchased from American Dye Source and its molecular mass is unknown. It was solved in toluene to obtain pure F8BT films; concentrations employed vary from 10 up to $30 \mathrm{~g} \mathrm{l}^{-1}$. All materials have been used as received without any further purification.
Dynamic spin-coating (spinner model SCV from Novocontrol) is performed over glass substrates in a laboratory environment without temperature or humidity control. Rotational speed spans in the range $60-150$ rotations per second (rps). When PVK is used, after each bilayer deposition, a 4 min baking step at $70{ }^{\circ} \mathrm{C}$ on a hot plate was performed in order to improve the optical quality of the films. Layer thickness was controlled by changing the spinning speed and solution concentrations. Note that the repeatability of single layer deposition is a crucial issue to obtain good quality DBRs.

Angle resolved transmittance ( $\mathrm{p}$ and s polarized), normal incidence reflectance and angle resolved photoluminescence spectra are measured with optical set-ups based on optical fiber coupled Avantes 2048 compact spectrometers working in the 200-1100 (350-1100) $\mathrm{nm}$ range having a spectral resolution of about 1.4 (1.6) nm. A combined deuterium-halogen light source Micropak DH-2000-BAL was used for transmittance and reflectance measurements. For photoluminescence (PL) experiments, an Oxxius (model 405-50-COL-PP) $405 \mathrm{~nm}$ continuous wave laser diode with a maximum power of $50 \mathrm{~mW}$ was employed. A Semrock notch filter (model LP02-442RS25) with a $450 \mathrm{~nm}$ cut-off wavelength is used to prevent laser light reaching the detector. PL is then recorded by the same spectrometers used for optical characterization. Additional details on the set-up can be found in [35].

Optical pumping of the microcavity is performed using an amplified Ti:sapphire laser system delivering $\sim 60$-fs pulses at $800-\mathrm{nm}$ central wavelength with a repetition rate of $1 \mathrm{kHz}$. Such pulses are frequency doubled (central wavelength at 400 $\mathrm{nm})$ in a $\beta$-barium borate crystal to match the absorption spectral region of F8BT and focused to a circular spot of $80 \mu \mathrm{m}$ diameter. Emission is collected by a spectrograph, with a resolution of $0.5 \mathrm{~nm}$ (Princeton Instruments sp-2156i, pixels CCD), through a bundle of optical fibers (19 fibers, $200 \mu \mathrm{m}$ diameter) positioned orthogonal to the microcavity at a distance $\sim 0.5 \mathrm{~cm}$ from it. The pump beam impinges at an angle of $\sim 45^{\circ}$ to the microcavity to avoid collection of the pump light in emission detection.

\section{Results and discussion}

Figure 1(a) shows the reflectance $(R)$ spectrum for a DBR made of 13 bilayers of cellulose acetate and poly(vinyl carbazole), CA:PVK, thickness $\sim 105 \mathrm{~nm}$ for CA and $\sim 96 \mathrm{~nm}$ for PVK. The $R$ spectrum of this DBR mirror shows the presence on an intense peak at about $630 \mathrm{~nm}$, and an anomalous structure is detected at about $350 \mathrm{~nm}$ where the carbazole group absorption is known to occur [36, 37], which masks the second-order gap of the photonic crystal, which is instead observed when polystyrene was used instead of PVK [28]. Moreover, the background of the spectrum is characterized by an extended pattern of interference fringes testifying the quality of the structure, despite the fact that it is obtained with a simple technique like spin-coating. Besides this general observation, a relevant comment concerns the polymer refractive index contrast and its relation to the bandwidth $(\Delta E)$ of the reflectance peak associated to the photonic band gap. Indeed, for a DBR in the $\lambda / 4$ condition, 

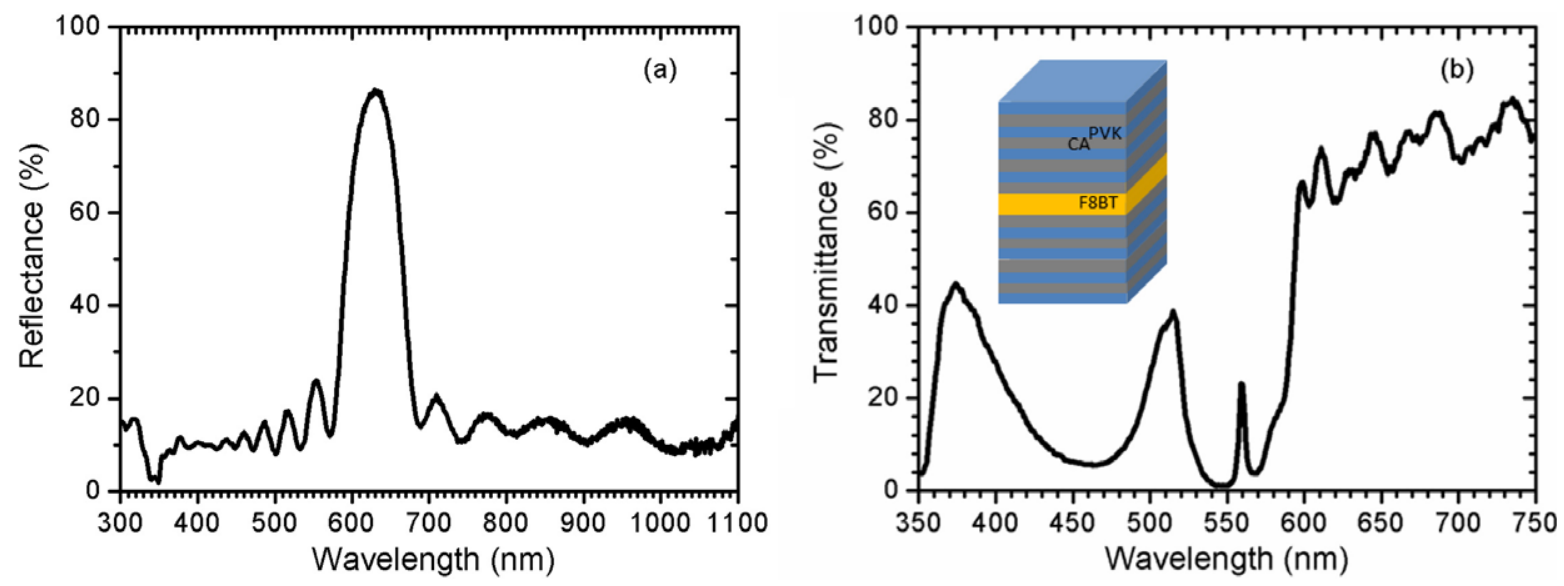

Figure 1. (a) Reflectance spectrum for a 13 CA:PVK bilayer DBR. (b) Transmittance spectra for a PVK:CA microcavity with a F8BT defect layer; inset: scheme of the microcavity structure.
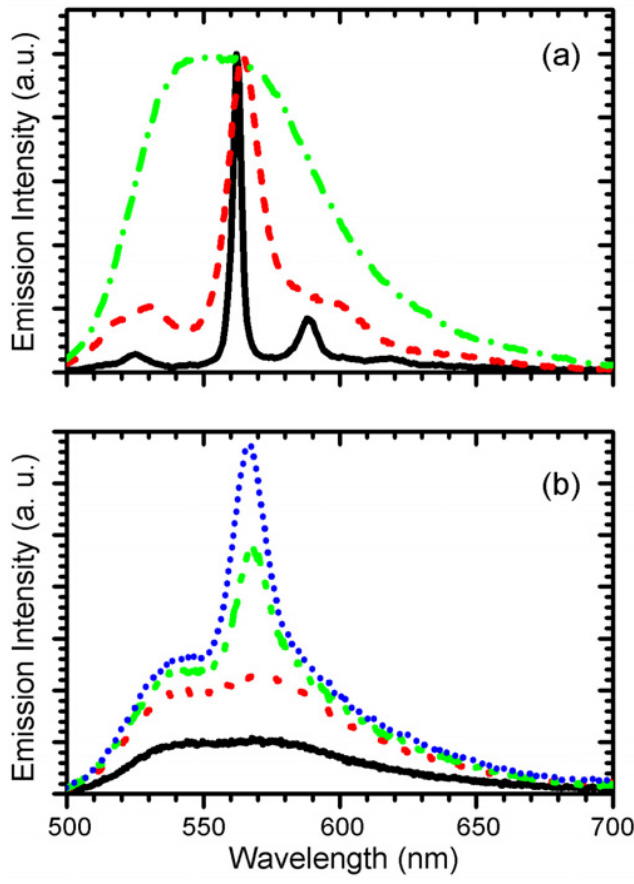

Figure 2. (a) Peak normalized CW emission spectra of PVK:CA (full black line), and PS:CA (dashed red line) microcavities containing an F8BT defect layer. The PL normalized spectrum of neat F8BT films is reported for comparison (dash-dotted, green line) (b) Emission spectra of neat F8BT film as a function of the pumping fluence (for 12, 27, 43 and $58 \mathrm{~mJ} \mathrm{~cm}^{-2}$, corresponding to black solid, red dashed, green dash-dotted and blue dotted lines, respectively).

the bandwidth is related to refractive indexes $\left(n_{1}, n_{2}\right)$ of composing media [38]:

$$
\Delta E=\frac{4}{\pi} E \sin ^{-1}\left(\frac{\left|n_{1}-n_{2}\right|}{n_{1}+n_{2}}\right) .
$$

In our previous letter, where polystyrene (PS) was used instead of PVK, a bandwidth of about $600-700 \mathrm{~cm}^{-1}$ was obtained [28], while in this case $\Delta E=1283 \mathrm{~cm}^{-1}$. This result indicates that PVK based structures allow one to better spatially confine a wider part of the PL spectrum. Moreover, the increased dielectric contrast allows one to achieve high reflectivity values with a lower number of bilayers than for DBR made with cellulose acetate and polystyrene [28].

In figure 1(b), the transmittance $(T)$ spectrum of a microcavity made by two 18 PVK:CA bilayer mirrors sandwiching an F8BT film is reported. The thickness of PVK, CA and F8BT layers is about 85, 95 and $135 \mathrm{~nm}$, respectively. The broad absorption band of F8BT shows a maximum at $\sim 460 \mathrm{~nm}$ and the photonic band gap is detected in the range 510-600 nm with the cavity mode, the sharp peak within the band gap, peaked at $564 \mathrm{~nm}$. In addition, a complicated interference pattern is observed on the background of the cavity $T$ spectrum, which is related to the thickness of the overall system. The thickness of the PVK and CA layers in the DBR mirrors was adjusted in order to obtain a photonic band gap overlapping the PL spectrum of F8BT, while the thickness of the F8BT cavity layer was finely tuned in order to match the defect mode spectral position with the amplified spontaneous emission peak of F8BT neat films (see below).

An additional important consequence of the high dielectric contrast in PVK:CA DBR concerns the microcavity quality factor $(Q=E / \Delta E=\lambda / \Delta \lambda)$. Figure 2 compares the peak normalized emission spectra under $\mathrm{CW}$ excitation of micro- cavities made with PVK:CA DBR mirrors and containing the F8BT defect layer with the corresponding ones where PVK is replaced by polystyrene as well as with the PL emission spectrum for a reference neat F8BT film. The microcavity redistributes the oscillator strength of the PL spectrum into a sharp emission peak possessing the directionality provided by the photonic crystal structure [28]. Data reported in fig- ure 2(a) show that a PS:CA microcavity, with an index contrast 1.59:1.45, has $Q$ around 43, comparable to that previously reported [28]. By using PVK:CA the refractive index contrast increases (1.68:1.45) thus boosting the confinement effects and then further increasing the PL enhancement. Indeed, as shown in figure 2(a), a FWHM of 5-7 nm is routinely achieved at $562 \mathrm{~nm}$ thus giving a $Q$ value in the range $80-100$. As far as PL intensity enhancement is concerned, PVK:CA microcavities show a peak intensity which is almost six times larger than that 

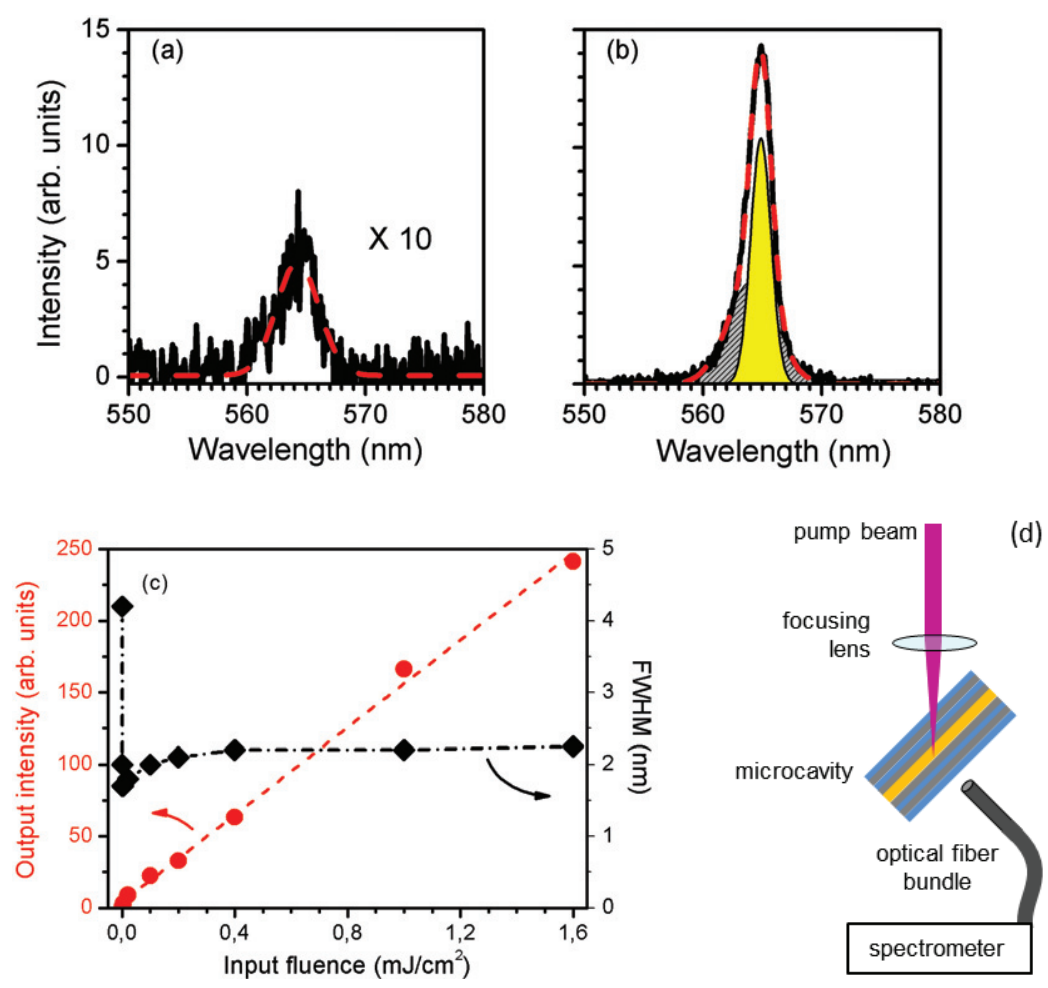

Figure 3. PVK:CA microcavity with the F8BT defect layer emission characteristics. (a) Emission spectrum for pump fluence below laser threshold (magnified by a factor of 10, black solid line) and corresponding Gaussian best fit (red dashed line); (b) emission spectrum for a pump fluence above laser threshold (black solid line) and corresponding best fit (red dashed line) with the two Gaussian components (gray, broad emission; yellow, lasing emission); (c) emission intensity (red circles), with linear fit (red dashed line), and FWHM of the emission spectra (black diamonds and dot-dashed line) as a function of the excitation fluence; (d) scheme of the experimental setup.

observed for the very same F8BT film. The enhancement is not affected by self-absorption since the spectral redistribution occurring due to the microcavity reduces the overlap between absorption and emission.

By checking different regions of the sample surface, we can find spots where sample homogeneity is particularly high and then the PL FWHM is further reduced to $3 \mathrm{~nm}$, thus increasing the quality factor above 180 (not shown here). Concerning sample inhomogeneity, we would like to note that the deposition of PVK and CA layers is not straightforward due to the non-perfect wettability of the surfaces, which can give rise to non-homogeneous films. The thermal annealing applied to the structure strongly improves the sample quality even though minor inhomogeneities are still retained. This indicates that there is room to further increase the optical quality of the full polymer microcavities, in particular for what concerning the deposition of PVK on CA.

Since the quality of the microcavity can be very high, such devices could be suitable to sustain laser action. First of all, we tested an F8BT neat reference film spin-coated on a glass substrate with the same deposition parameters used for the defect layer of our microcavity. Under pulsed optical pumping we observe in plane amplified spontaneous emission (ASE)(figure 2(b)): indeed, upon increasing the pump power, a peak comes out from the PL background centered at $\sim 567$ $\mathrm{nm}$, having a full width half maximum of $19 \mathrm{~nm}$. These data are in full agreement with previous reports on the same conjugated polymer $[39,40]$.
Pulsed optical pumping was then applied to our full polymer microcavities possessing a very high $Q$ factor and spectrally tuned to the ASE peak. Results are summarized in figure 3.

At low excitation fluences, only the broad photoluminescence signal can be detected, as shown in figure 3(a). This PL spectrum can be well fitted with a Gaussian curve (centered at 564,2 nm) with a FWHM of $4.2 \mathrm{~nm}(Q=134)$, in agreement with the transmission spectrum of the microcavity and on its PL spectrum under CW pumping. For pump fluences higher than a certain threshold value (figure 3(b)), the emission spectrum shows an asymmetric shape and can be well fitted (red dashed line) only using two Gaussian curves, the first centered at around $564 \mathrm{~nm}$ with a FWHM around $4.4 \mathrm{~nm}$ while the second, more intense, with peak centered at $564.9 \mathrm{~nm}$ and possessing a FWHM $<2.2 \mathrm{~nm}$. In figure $3(\mathrm{~b})$ we display the emission spectrum obtained on pumping just above threshold, where the higher intensity Gaussian has a FWHM of $1.7 \mathrm{~nm}$ (yellow area). We assign this high intensity sharp Gaussian component of the spectrum to a single mode laser emission from the cavity. The lower intensity broader component (light gray area) is assigned to the PL, which is possibly emitted in a direction non orthogonal to the cavity and which is detected due to the relatively large collection optics in our setup together with the low divergence laser beam. The lasing assignment is supported by the emission characteristics, i.e. the output intensity and the FWHM of the high intensity sharp component as a function of the input fluence (figure 3(c)). We observe a 
clear line narrowing effect joined to a linear dependence of the output intensity as a function of the input fluence for fluences higher than the threshold value in agreement with standard expectation for lasing emission. No other emission processes such as PL or ASE would show similar line narrowing or linear behavior [41].

From data in figure 3(c), we also notice that after the dramatic narrowing at threshold, the FWHM slightly increases upon increasing the excitation fluence. This behavior is not an artifact but is typical for a laser working in the gain switching regime [41] as already reported in literature for organic lasers [30]. As a matter of fact, when short pulses are used to create the population inversion, the avalanche process depopulating the excited state during laser action occurs in shorter times as the excited population increases. As a result, as the energies of the pump pulses increase, the emitted pulses show a reduction in duration and, correspondingly, an increase in the width of their spectrum. Finally, from the data just above threshold, we estimate a coherence length [42] $\xi_{\text {coh }}=\lambda_{\text {laser }}^{2} / \Delta \lambda_{\text {laser }} \sim 0.2 \mathrm{~mm}$.

An important property of our all plastic microcavity is related to the laser threshold. Note that organic materials, despite the continuous band-like behavior of the HOMO and LUMO levels due to the large number of vibrational modes coupled to the electronic transition, to intermolecular interactions and to the distribution of conjugation lengths, behave as four-level systems [4] and, from laser rate equations in the four-level space independent approximation [41], threshold condition is achieved when the gain equals the losses, namely: $\sigma N l=\gamma$ where $\sigma$ is the cross section of stimulated emission, $N$ is the upper level population, $l$ is the length of the active material and $\gamma$ are the logarithmic losses. All the losses, regardless of their origin, are related to the quality factor of the cavity [41] and determine the lifetime of photons in the cavity $\tau_{\mathrm{c}}\left(Q=2 \pi v \tau_{\mathrm{c}}\right.$, with $v$ being the frequency of oscillation of the cavity), which in turn is related to $\gamma$ through the relation: $\gamma=t_{\mathrm{tr}} / \tau_{\mathrm{c}}$, where $t_{\mathrm{tr}}$ is the transit time of photons in the cavity $\left(t_{\mathrm{tr}}=n l / c\right.$ with $n$ being the index of refraction of the active material, and $c$ the speed of light in vacuum). In our microcavity $l$ is about $135 \mathrm{~nm}$, the index of refraction is 1.69 [39], so the transit time is $7.6 \times 10^{-16} \mathrm{~s}$; $v$ at $564 \mathrm{~nm}$ is $532 \mathrm{THz}$ and $Q$ is 134 , so $\tau_{\text {c }}$ turns out to be $4.0 \times$ $10^{-14}$ s. Being $\sigma=7.09 \times 10^{-16} \mathrm{~cm}^{2}$ [39], $N l$ is $2.66 \times 10^{13}$ $\mathrm{cm}^{-2}$, and the corresponding pump fluence absorbed by the F8BT film is $F=N l h v_{\mathrm{p}}=13 \mu \mathrm{J} \mathrm{cm}^{-2}$, with $h$ being Planck's constant and $v_{\mathrm{p}}$ the frequency of the pump photons $(750 \mathrm{THz})$. By taking into account the absorption of the film and reflectivity losses at $v_{\mathrm{p}}$ for the first DBR mirror, we find that only $64 \%$ of the photons impinging on the microcavity are absorbed by the F8BT, thus setting for the incident fluence a conservative threshold value of about $20 \mu \mathrm{J} \mathrm{cm}^{-2}$.

The accuracy in the measurement of the experimental laser threshold at very low power range corresponds to an error bar comparable to the dimension of the symbols in figure 3(c). A very conservative assumption provides a value lower than $20 \mu \mathrm{J} \mathrm{cm}^{-2}$, in agreement with the one calculated. Note that the threshold for laser in the microcavity and ASE in the film cannot be compared due to the different geometries used for investigating the two processes. Note also that we found a very low laser threshold value as compared to the best ones reported in literature for microcavities containing an organic material as the active layer. Lower thresholds have been so far obtained only by using high reflectance $(R>98 \%)$ commercial inorganic DBRs [32, 33], which are however not suitable for practical applications. Indeed, for future photonic devices, such as board-to-board and chip-tochip interconnects, with a capability of high integration and manufacturing onto soft matter, direct fabrication of DBRs is preferred. In this contest, the best results so far achieved have been for porous DBRs made with $\mathrm{TiO}_{2}$ and $\mathrm{SiO}_{2}$ colloids [10, $30,31]$.

In this letter, we demonstrate that our strategy allows us to make an all plastic device with the additional advantage of a low laser threshold. Notice also that the conjugated polymer active material does not require to be embedded in an inert polymer matrix (like for dyes or nanocrystals [22, 24, 25, 27, 29]) thus reducing negative effects related to phase segregation and/or scattering. Moreover, additional observations can be done by comparing microcavities having plastic DBR and gain medium composed of polymer matrices loaded with molecules/nanocrystals [24, 27, 29]. Even though the performances of the microcavities are mainly governed by the number of layers and their refractive index contrast [43], we notice that our full polymer microcavities show both a $Q$ factor and a laser threshold improved with respect to those reported $\left(8-30 \mathrm{~mJ} \mathrm{~cm}^{-2}\right)$ for a similar system where the emitter is a molecule dispersed (1-2\% by weight) into a polymer having a thickness in the range 4-350 $\mu \mathrm{m}[26,29]$. Only microcavities prepared by co-extrusion with a very large number of layers (more than 100) might achieve laser thresholds comparable to ours (35-435 $\left.\mu \mathrm{J} \mathrm{cm}^{-2}\right)$ [18, 44]. In our case the thickness of the cavity is reduced (about $135 \mathrm{~nm}$ ) and allows a good matching between the pumped region and the cavity mode inside the gain medium. Due to large absorption coefficients of polymers $\left(\alpha \approx 10^{5} \mathrm{~cm}^{-1}\right)$ [39] the penetration depth of the pump is of the order of a pair of hundreds of nanometers, and we thus create population inversion all along the cavity. The photons that travel back and forth between the two DBRs experience gain all along their path. We thus maximize the gain and minimize the losses. A similar approach is expected in the microcavity described by Menon et al [25] where the molecular gain medium is replaced by nanocrystals and the thickness of the cavity embedding them is $190 \mathrm{~nm}$; here the lasing threshold can be estimated (due to the incomplete data reported) to be at least tens of $\mathrm{mJ} \mathrm{cm} \mathrm{cm}^{-2}$ [25].

Let us make some additional considerations on the $Q$ factor. In our microcavity we have high $Q$-factor, and thus high $\tau_{\mathrm{c}}$, and a small $\tau_{\mathrm{tr}}$, due to the thin defect layer, and we thus reduce the losses $\left(\gamma=t_{\mathrm{tr}} / \tau_{\mathrm{c}}\right)$. This is an additional advantage of the choice of a thin gain region. On the basis of all such reasoning, we suggest that the use of fluorescent conjugated polymers as the cavity active layer could represent a significant improvement for the all plastic microcavity laser technology since they possess strong absorption and low losses when prepared as thin films. These properties cannot be achieved by molecular fluorophores or nanocrystals since as soon as they are assembled in neat films, intermolecular 
interactions quench the fluorescence. In turn, this observation justifies the dispersion of molecular/nanocrystals into a thick polymer matrix, which guarantees both molecule/nanocrystal separation and suppression of the quenching. However, the drawback of this approach is the requirement of a larger film thickness (due to the reduced density of the active material), which increases the volume cavity thus decreasing the confinement effect.

Finally, we mention a technological consideration about all polymer microcavities. It is worth noting that the threshold value we measured, corresponding to energies of less than one nanojoule per pulse, would allow one to operate in the regime of tens of $\mathrm{MHz}$ without causing material degradation (which has been estimated to occur in a polymer slab under $\mathrm{CW}$ excitation to be about $100 \mathrm{MW} \mathrm{cm}^{-3}$ ) [31]. Note that in this regime, even if the repetition rate is high, triplet state accumulation is not an issue since lasing action implies full depopulation of the excited state in a time (of the order of a few picoseconds [40]) shorter than the typical time required to populate triplet states in conjugated polymers (about $10^{-6}-10$ -7 s) [45]. A further favorable result of the microcavity concerns the stability under excitation; in fact, we could not observe appreciable degradation of the emission after tens of minutes of operation, working in ambient conditions.

\section{Conclusions}

In conclusion, we showed that all polymer flexible microcavities prepared by using PVK and CA DBRs and containing an F8BT emitting layer can be finely tuned in order to have the defect mode spectrally overlapped with the ASE peak of the neat F8BT film. For these tuned microcavities, laser action is achieved, under pulsed optical pumping. These microcavities possess a laser threshold which is low and which is lower than the ones obtained either with organic active material and inorganic DBRs or with molecular/nanocrystal gain materials embedded into a polymer matrix and organic DBRs. The all plastic microcavity laser thus represents the best strategy for future compact, soft and low-cost devices. Note also that the combination of the capability of integration, typical of vertical surface-emitting devices, and of the fabrication of soft devices onto soft substrates can be a keystone for future photonic applications for low-cost scale-up manufacturing devices.

\section{Acknowledgments}

The work in Genova was partially supported by the Ministry of the University and Scientific and Technological Research through the PRIN project 2010XLLNM3 (2010-2011). Within such project, a formal collaboration agreement between the University of Genoa and CNST-IIT has been established. Margherita Zavelani-Rossi acknowledges Fondazione Cariplo for financial support through grant no. 2012-0824.

\section{References}

[1] Moses D 1992 Appl. Phys. Lett. 603215

[2] Chenais S and Forget S 2012 Polym. Int. 61390
[3] Samuel I D W, Namdas E B and Turnbull G A 2009 Nature Photon. 3546

[4] Clark J and Lanzani G 2010 Nature Photon. 4438

[5] McGehee M D and Heeger A J 2000 Adv. Mater. 121655

[6] Tocci M D, Scalora M, Bloemer M J, Dowling J P and Browden C M 1996 Phys. Rev. A 532799

[7] Tessler N, Denton G J and Friend R H 1996 Nature 382695

[8] Hayes G R, Cacialli F and Phillips R T 1997 Phys. Rev. B 56 4798

[9] Schulzgen A, Spiegelberg C, Morrell M M, Mendes S B, Kippelen B, Peyghambarian N, Nabor M F, Mash E A and Allemand P M 1998 Appl. Phys. Lett. 72269

[10] Persano L, Camposeo A, Carro P D, Mele E, Cingolani R and Pisignano D 2006 Appl. Phys. Lett. 89121111

[11] Kena C S and Forrest S R 2010 Nature Photon. 4371

[12] Brückner R, Sudzius M, Hintschich S I, Fröb H, Lyssenko V G and Leo K 2011 Phys. Rev. B 83033405

[13] Bruckner R, Zakhidov A A, Scholz R, Sudzius M, Hintschich S I, Frob H, Lyssenko V G and Leo K 2012 Nature Photon. 6322

[14] Mazzeo M, della Sala F, Mariano F, Melcarne G, D'Agostino S, Duan Y, Cingolani R and Gigli G $2010 \mathrm{Ad} v$. Mater. 224696

[15] Somaschi N, Mouchliadis L, Coles D, Perakis I E, Lidzey D G, Lagoudakis P G and Savvidis P G 2011 Appl. Phys. Lett. 99143303

[16] Virgili T, Coles D, Adawi A M, Clark C, Michetti P, Rajendran S K, Brida D, Polli D, Cerullo G and Lidzey D G 2011 Phys. Rev. B 83245309

[17] Sandrock M, Wiggins M, Shirrk J S, Tai H, Ranade A, Baer E and Hiltner A 2004 Appl. Phys. Lett. 84362

[18] Song H, Singer K, Lott J, Wu Y, Zhou J, Andrews J, Baer E, Hiltner A and Weder C 2009 J. Mater. Chem. 197520

[19] Katouf R, Komikado T, Itoh M, Yatagai T and Umegaki S 2005 Photon. Nanostruct. 3116

[20] Álvarez A L, Tito J, Vaello M B, Velásquez P, Mallavia R, S ánchez-López M M and Fernández de Ávila S 2003 Thin Solid Films $\mathbf{4 3 3} 277$

[21] Fink Y, Winn J N, Fan S, Chen C, Michel J, Joannopoulos J D and Thomas E L 1998 Science 2821679

[22] Komikado T, Yoshida S and Umegaki S 2006 Appl. Phys. Lett. 89061123

[23] Scotognella F, Monguzzi A, Cucini M, Meinardi F, Comoretto D and Tubino R 2008 Int. J. Photoenergy 2008 389034

[24] Valappil N V, Luberto M, Menon V M, Zeylikovich I, Gayen T K, Franco J, Das B B and Alfano R R 2007 Photon. Nanostruct. 5184

[25] Menon V M, Luberto M, Valappil N V and Chatterjee S 2008 Opt. Express 1619535

[26] Sakata H, Takeuchi H, Natsume K and Suzuki S 2006 Opt. Express 1411681

[27] Takeuchi H, Natsume K, Suzuki S and Sakata H 2007 Electron. Lett. 4330

[28] Frezza L, Patrini M, Liscidini M and Comoretto D $2011 \mathrm{~J}$. Phys. Chem. C 11519939

[29] Goldenberg L M, Lisinetskii V and Schrader S 2013 Laser Phys. Lett. 10055808

[30] Persano L, Carro P D, Mele E, Cingolani R, Pisignano D, Zavelani-Rossi M, Longhi S and Lanzani G $2006 \mathrm{Appl}$. Phys. Lett. 88121110

[31] Scotognella F, Puzzo D P, Zavelani-Rossi M, Clark J, Sebastian M, Ozin G A and Lanzani G 2011 Chem. Mater. 23805 
[32] Granlund T, Theander M, Berggren M, Andersson M, Ruzeckas A, Sundström V, Björk G, Granström M and Inganäs O 1998 Chem. Phys. Lett. 288879

[33] Lee T-W, Park O O, Cho H N, Kim D Y and Kim Y C 2003 J. Appl. Phys. 931367

[34] Scotognella F, Monguzzi A, Meinardi F and Tubino R 2010 Phys. Chem. Chem. Phys. 12337

[35] Berti L, Cucini M, Di Stasio F, Comoretto D, Galli M, Marabelli F, Manfredi N, Marinzi C and Abbotto A 2010 J. Phys. Chem. C 1142403

[36] Colombi C, Comoretto D, Cuniberti C, Musso G, Piaggio P, Dellepiane G, Novi M, DellErba C and Bolognesi A 1996 Macromol. Chem. Phys. 1971241

[37] Comoretto D, Dellepiane G, Cuniberti C, Rossi L, Borghesi A and Le Moigne J 1996 Phys. Rev. B 5315653
[38] Joannopulos J D, Meade R D and Win J N 1995 Photonic Crystals: Molding the Flow of the Light (Princeton, NJ: Princeton University Press)

[39] Xia R, Heliotis G, Hou Y and Bradley D D C 2003 Org. Electron. 4165

[40] Zavelani-Rossi M, Perissinotto S, Lanzani G, Salerno M and Gigli G 2006 Appl. Phys. Lett. 89181105

[41] Svelto O 1998 Principles of Lasers 4th edn (New York: Springer)

[42] Coles H and Morris S 2010 Nature Photon. 4676

[43] Saleh B E A and Teich M C 2007 Fundamentals of Photonics (Hoboken, NJ: Wiley)

[44] Song H et al 2009 Proc. SPIE 7467 74670A

[45] Yu J, Lammi R, Gesquiere A J and Barbara P F 2005 J. Phys. Chem. B 10910025 